\title{
Acute Hypokalemia May Not Be an Effective Way to Sensitize the In Situ Canine Heart for Sparfloxacin-Induced Long QT Syndrome
}

\author{
Katsuyoshi Chiba $^{1}$, Atsushi Sugiyama ${ }^{2, *}$, Kazuo Watanabe ${ }^{1}$, Kiyoshi Takasuna ${ }^{1}$, and Keitaro Hashimoto ${ }^{2}$ \\ ${ }^{1}$ New Product Research Laboratories II, Daiichi Pharmaceutical Co., Ltd., \\ 16-13, Kita-Kasai 1-chome, Edogawa-ku, Tokyo 134-8630, Japan \\ ${ }^{2}$ Department of Pharmacology, Interdisciplinary Graduate School of Medicine and Engineering, University of Yamanashi, \\ Tamaho-cho, Nakakoma-gun, Yamanashi 409-3898, Japan
}

Received August 11, 2005; Accepted November 21, 2005

\begin{abstract}
Extents of the sparfloxacin (3-10 mg/kg, i.v.)-induced QT interval prolongation under normokalemic and hypokalemic conditions were assessed in halothane-anesthetized beagle $\operatorname{dogs}(\mathrm{n}=5)$. The hypokalemic condition was induced by an oral administration of furosemide ( $200 \mathrm{mg} / \mathrm{kg}$ per day) for 3 days, which decreased the serum potassium concentration from $3.65 \pm 0.13$ to $2.35 \pm 0.13 \mathrm{mM}(P<0.05)$. However, the decrease of potassium concentration by itself did not affect the extent of the sparfloxacin-induced QT interval prolongation. These results indicate that acute hypokalemia may not severely sensitize the in situ heart for drug-induced long QT syndrome as previously thought.
\end{abstract}

Keywords: hypokalemia, long QT syndrome, fluoroquinolone

Non-cardiovascular drug-induced QT interval prolongation is often associated with the onset of torsades de pointes (TdP) a life-threatening ventricular arrhythmia (1). Sparfloxacin, a fluoroquinolone antibacterial agent, has been reported to prolong the QT interval and to cause TdP in both experimental and clinical studies via an inhibition of ether-à-go-go-related gene (HERG) mediated $\mathrm{K}^{+}$currents $(2-5)$. Recently, development of new animal models that can predict the risk of the drug-induced long QT syndrome has been strongly encouraged by the ICH S7B guideline (6). In this study, we assessed the effects of acute hypokalemia on the electropharmacological profile of sparfloxacin in halothane-anesthetized dogs since hypokalemia has been shown to enhance proarrhythmic potentials of QTprolonging drugs $(7,8)$.

All experiments were performed in accordance with the Guidelines for Animal Experiments, Daiichi Pharmaceuticals Co., Ltd. and University of Yamanashi. Experiments were carried out using female beagle dogs weighing about $10 \mathrm{~kg}(\mathrm{n}=5)$. The electropharmacological effects of sparfloxacin were assessed in the

*Corresponding author. atsushis@yamanashi.ac.jp

Published online in J-STAGE: January 11, 2006

DOI: 10.1254/jphs.SCJ05008X normokalemic condition (Experiment 1) followed by the hypokalemic condition (Experiment 2) in series, as depicted in Fig. 1, which can reduce the possible variability of the results among the individuals.

In each experiment, dogs were initially anesthetized with thiopental sodium $(30 \mathrm{mg} / \mathrm{kg}$, i.v.). After intubation, $1 \%$ halothane vaporized with $100 \%$ oxygen was inhaled with a ventilator (SN-480-3; Shinano, Tokyo). Tidal volume and respiratory rate were set at $20 \mathrm{ml} / \mathrm{kg}$ and 15 strokes $/ \mathrm{min}$, respectively. The systemic blood pressure was continuously monitored at the right femoral artery. The surface lead II ECG was obtained from the limb electrodes. A bi-directional steerable monophasic action potential (MAP) recording/pacing combination catheter (1675P; EP Technologies, Sunnyvale, CA, USA) was positioned at the endocardium of the interventricular septum in the right ventricle through the right femoral vein to obtain MAP signals. The duration of the MAP signals was measured at $90 \%$ repolarization level $\left(\mathrm{MAP}_{90}\right)$. The heart was electrically driven using a cardiac stimulator (SEC3102; Nihon Kohden, Tokyo) with the pacing electrodes of the MAP recording/pacing combination catheter placed in the right ventricle. Stimulation pulses were rectangular in shape, $2 \mathrm{~V}$ of amplitude, and $1 \mathrm{~ms}$ of 

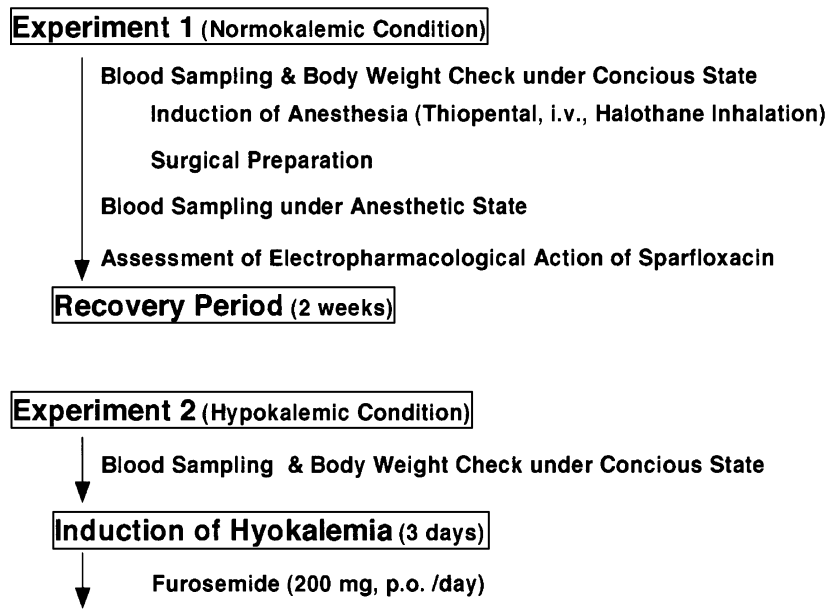

\begin{tabular}{|l} 
Blood Sampling \& Body Weight Check under Concious State \\
Induction of Anesthesia (Thiopental, i.v., Halothane Inhalation) \\
Surgical Preparation \\
Blood Sampling under Anesthetic State \\
Assessment of Electropharmacological Action of Sparfloxacin
\end{tabular}

Fig. 1. Experimental protocol. The electropharmacological effects of sparfloxacin were assessed in the normokalemic condition (Experiment 1) followed by the hypokalemic condition (Experiment 2) in series.

duration. The $\mathrm{MAP}_{90}$ was measured during the sinus rhythm $\left(\mathrm{MAP}_{90(\text { (sinus) }}\right)$ and at pacing cycle lengths of $400 \mathrm{~ms}\left(\mathrm{MAP}_{90(\mathrm{CL} 400)}\right)$ and $300 \mathrm{~ms}\left(\mathrm{MAP}_{90(\mathrm{CL} 300)}\right)$. The effective refractory period (ERP) of the right ventricle was assessed by programmed electrical stimulation. The pacing protocol consisted of 5 beats of basal stimuli in a cycle length of $400 \mathrm{~ms}$ followed by an extra stimulus of various coupling intervals. Starting from the late diastole, the coupling interval was shortened in 5-ms decrements until refractoriness occurred. The terminal repolarization period $\left(\mathrm{TRP}=\mathrm{MAP}_{90(\mathrm{CL} 400)}-\mathrm{ERP}\right)$ of the right ventricle was calculated to estimate the duration of phase 3 repolarization of action potential, which would reflect the extent of ventricular electrical vulnerable period $(9,10)$. In addition, the increment of $\mathrm{MAP}_{90}$ was calculated for the pacing cycle length of 300 and $400 \mathrm{~ms}$ to analyze the reverse use-dependent prolongation of repolarization. The parameters were continuously monitored using a polygraph system (RM-6000, Nihon Kohden) and analyzed with an ECG processor (SBP-8; Softron, Tokyo). Corrected QT interval (QTc) was obtained using Van de Water's formula (11). The values of ECG parameters and $\mathrm{MAP}_{90}$ represent the mean of three consecutive complexes at each time point of assessment.

In Experiment 1, the venous blood in a volume of $2 \mathrm{ml}$ was initially sampled before the induction of anesthesia. After confirming the stability of the cardiohemodynamic condition under the anesthetic state, the pre-drug control assessment was performed. Then, the venous blood in a volume of $2 \mathrm{ml}$ was sampled again under the anesthetic state. Next, $3 \mathrm{mg} / \mathrm{kg}$ of sparfloxacin, which provides clinically relevant antibiotic plasma concentration of $1-2 \mu \mathrm{g} / \mathrm{ml}$ (4), was administered over $10 \mathrm{~min}$ into the right femoral vein and each cardiovascular variable was recorded at 5, 10, 20, and $30 \mathrm{~min}$ after the start of infusion. Finally, a supratherapeutic dose of $10 \mathrm{mg} / \mathrm{kg}$ of sparfloxacin was additionally administered over $10 \mathrm{~min}$ and each variable was recorded in the same manner. After a recovery period of two weeks, a diuretic drug, furosemide $(200 \mathrm{mg} / \mathrm{kg}$ per day), was orally administered for 3 days consecutively to induce hypokalemia $(12,13)$. Then, in Experiment 2, the same experimental protocol as that in Experiment 1 was repeated.

The serum sodium and potassium concentrations in each blood sample were analyzed using an automatic electrolyte counter (710, Hitachi, Tokyo), whereas the creatinine concentration was determined using an automatic analyzer (7350, Hitachi). Sparfloxacin was extracted from the commercial source (SparaTM; Dainippon, Tokyo), which was dissolved in $0.5 \%$ lactate solution in concentrations of 2.0 and $6.7 \mathrm{mg} / \mathrm{ml}$. The solutions were prepared freshly for each experiment. The following drugs were purchased: thiopental sodium (Tanabe, Osaka), halothane (Takeda, Osaka), and furosemide (Sigma, St. Louis, MO, USA). Data are presented as the mean \pm S.E.M. The statistical differences within a parameter were evaluated by the paired $t$-test or one-way, repeated-measures analysis of variance (ANOVA) followed by Contrasts for mean values comparison. A $P$-value $<0.05$ was considered significant.

The effects of furosemide on the serum sodium, potassium, and creatinine concentrations and the body weight are summarized in Table $1(n=5)$. Meanwhile, the time courses of changes in the heart rate, mean blood pressure, QT interval, QTc, MAP ${ }_{90(\text { sinus }), M} \mathrm{MAP}_{90(\mathrm{CL} 400)}$, $\mathrm{MAP}_{90(\mathrm{CL} 300)}$, ERP, and TRP are summarized in Fig. 2 $(n=5)$. Administration of furosemide decreased the serum potassium concentration and body weight, whereas no significant change was detected in the sodium or creatinine concentration. Potassium concentration decreased further after the induction of anesthesia in both Experiments 1 and 2, whereas no significant change was detected in the sodium or creatinine concentration. The mean blood pressure in Experiment 2 was significantly lower than that in Experiment 1, whereas no significant difference was detected in any 
Table 1. Comparison of biochemical data and body weight $(n=5)$

\begin{tabular}{llccccc}
\hline Experiment No. & Condition & Anesthesia & $\mathrm{Na}^{+}(\mathrm{mM})$ & $\mathrm{K}^{+}(\mathrm{mM})$ & Creatinine $(\mathrm{mg} / \mathrm{ml})$ & Body weight $(\mathrm{kg})$ \\
\hline Experiment 1 & (Normokalemia) & $(-)$ & $148.9 \pm 0.6$ & $4.35 \pm 0.20$ & $0.63 \pm 0.04$ & $11.0 \pm 0.2$ \\
& (Normokalemia) & $(+)$ & $148.8 \pm 0.8$ & $3.65 \pm 0.13^{\#}$ & $0.62 \pm 0.03$ & \\
Experiment 2 & Pre-furosemide & $(-)$ & $146.6 \pm 2.6$ & $4.36 \pm 0.06$ & $0.67 \pm 0.02$ & $10.6 \pm 0.2$ \\
& (Hypokalemia) & $(-)$ & $139.4 \pm 1.2$ & $3.03 \pm 0.03^{*}$ & $0.77 \pm 0.04$ & $10.0 \pm 0.1^{*}$ \\
& (Hypokalemia) & $(+)$ & $137.9 \pm 2.0$ & $2.35 \pm 0.13^{* \# \#}$ & $0.72 \pm 0.03$ & \\
\hline
\end{tabular}

Data are presented as the mean \pm S.E.M. ${ }^{*} P<0.05$, compared with the respective normokalemic values. ${ }^{\sharp} P<0.05$, compared with the respective non-anesthetized condition values.
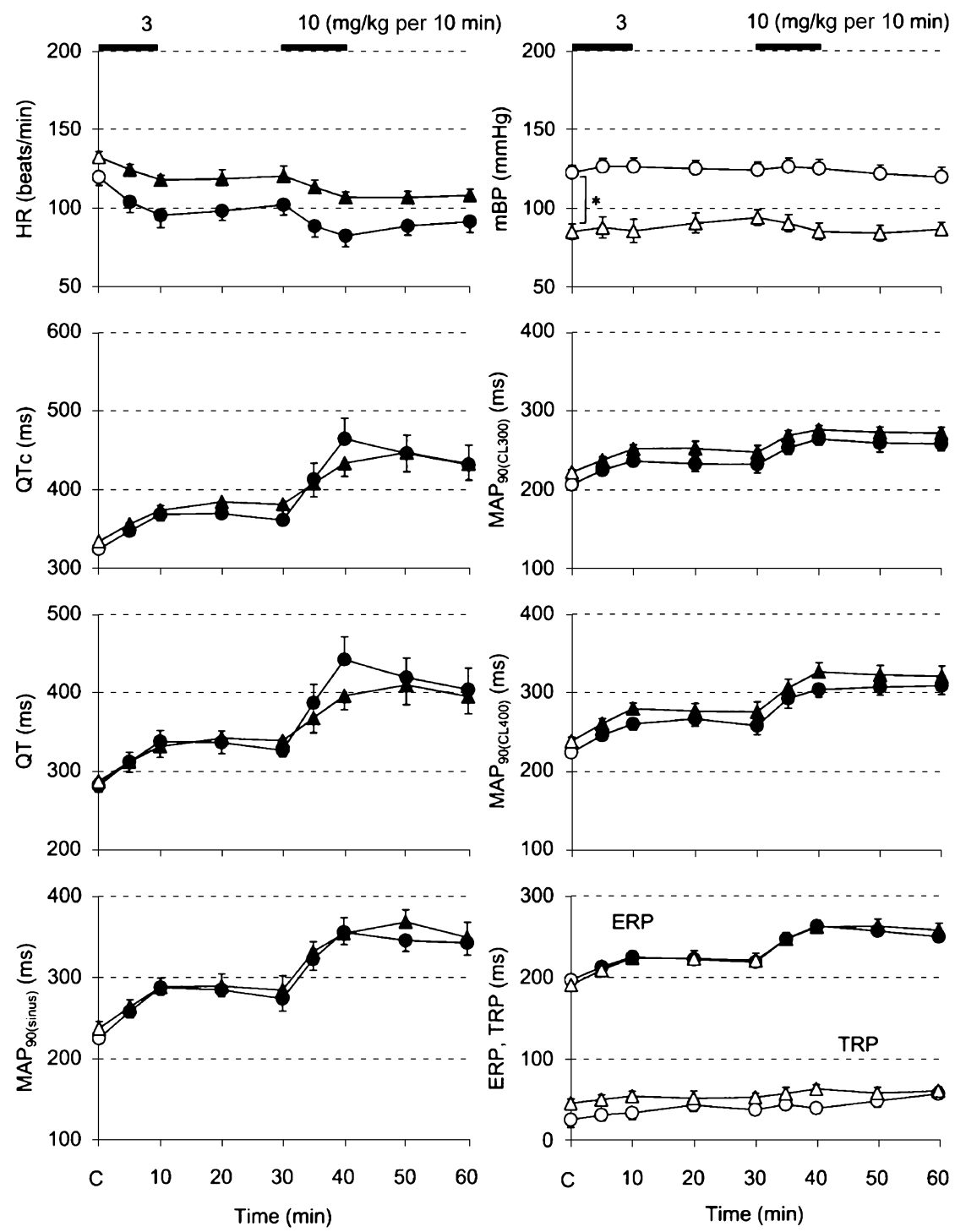

Fig. 2. Time courses of the heart rate (HR), mean blood pressure (mBP), QT interval (QT), QTc, MAP ${ }_{90}$, effective refractory period (ERP), and terminal repolarization period $\left(\mathrm{TRP}=\mathrm{MAP}_{90(\mathrm{CL} 400)}-\mathrm{ERP}\right)(\mathrm{n}=5$ for each group). Sparfloxacin was intravenously infused for $10 \mathrm{~min}$ under the normokalemic (Experiment 1, circles) or hypokalemic (Experiment 2, triangles) condition. $\mathrm{MAP}_{90}$ represents the duration of the monophasic action potential at $90 \%$ repolarization level. $\mathrm{MAP}_{90(\text { sinus) }}$ : $\mathrm{MAP}_{90}$ during the sinus rhythm. $\mathrm{MAP}_{90(\mathrm{CL} 400)}$ and $\mathrm{MAP}_{90(\mathrm{CL} 300)}$ : $\mathrm{MAP}_{90}$ at pacing cycle lengths of 400 and $300 \mathrm{~ms}$, respectively. Data are presented as the mean \pm S.E.M. The closed symbols represent the significant differences from the respective control values (C) by $P<0.05 . * P<0.05$. 
of the other parameters between Experiments 1 and 2 . In Experiment 1, intravenous administration of sparfloxacin decreased the heart rate in a dose-related manner, whereas no significant change was detected in the mean blood pressure. It also prolonged the QT interval, QTc, $\mathrm{MAP}_{90(\text { (sinus) }}, \mathrm{MAP}_{90(\mathrm{CL} 400)}, \mathrm{MAP}_{90(\mathrm{CL} 300)}$, and ERP in a dose-related manner, whereas TRP was hardly affected. The extent of the drug-induced prolongation of the $\mathrm{MAP}_{90}$ was more prominent at slower pacing rate. On the other hand, in Experiment 2, the same electropharmacological profile of sparfloxacin as that observed in Experiment 1 was confirmed. Neither ventricular arrhythmia nor early afterdepolarization was observed during the whole experimental period.

The present study was designed to assess effects of acute hypokalemia on the electropharmacological profile of sparfloxacin using the well-established halothane-anesthetized canine model $(3,4)$. As shown in Experiment 1, therapeutic to supratherapeutic doses of sparfloxacin decreased the heart rate without affecting the mean blood pressure and prolonged the MAP duration and ERP to a similar extent, resulting in no change in the TRP. The extent of prolongation of $\mathrm{MAP}_{90}$ was more prominent at slower pacing rate, indicating that sparfloxacin can delay the ventricular repolarization in a reverse use-dependent manner, which may reflect characteristics of the $\mathrm{I}_{\mathrm{Kr}}$-channel-blocking property of the drug (2). Thus, the negative chronotropic action of sparfloxacin itself may enhance the prolongation of the ventricular repolarization process. This electropharmacological profile of sparfloxacin was in good accordance with our previous study of sparfloxacin (4), indicating the reliability and reproducibility of our experimental system.

As shown in Experiment 2, furosemide-induced acute hypokalemia did not modulate the electropharmacological profile of sparfloxacin at all, which is essentially in accordance with the previous in vivo studies describing that hypokalemia is a less important factor for the onset of class III drug-induced $\operatorname{TdP}(12,13)$. On the other hand, a previous in vitro study indicated that extracellular potassium plays an important role in determining the $\mathrm{I}_{\mathrm{Kr}}$-blocking potency of QT-intervalprolonging drugs (14); thus, further study is required to bridge the gap between the in vitro and in vivo findings. As in the case of the current in vivo experiment, furosemide-induced hypotension may have increased the sympathetic nervous tone on the heart, resulting in the increase of outward currents including $\mathrm{I}_{\mathrm{Ks}}$ and/or $\mathrm{I}_{\mathrm{Cl}}$, which might counterbalance the effect of hypokalemia on the repolarization period.

The effects of the induction of general anesthesia on the potassium concentration also deserve a comment.
As shown in Table 1, serum potassium concentration was reduced by $0.7 \mathrm{mM}$ after the induction of anesthesia in both Experiments 1 and 2, which is in good accordance with a clinical report (15). Also in a preliminary experiment $(n=7)$, we have assessed the effects of pentobarbital-anesthesia on the serum potassium concentration in $\operatorname{dogs}$ and found that the potassium concentration was reduced in a similar extent to that observed by halothane-anesthesia. These may be critical new findings in analyzing drug-induced QT interval prolongation of anesthetized animal models. Although the ionic mechanism that can explain the causal link between the decrease of potassium concentration and these anesthetics needs to be clarified, one can speculate that mechanical ventilation may also partly contribute to the reduction of potassium concentration by decreasing blood $\mathrm{CO}_{2}$ gas tension, namely, respiratory alkalosis.

In conclusion, acute hypokalemia may not increase the proarrhythmic risks of in situ intact heart for druginduced long QT syndrome, which may also indicate the importance of developing pathologically modified proarrhythmic models in predicting risks in humans during the nonclinical testing of new drugs.

\section{Acknowledgments}

This study was supported in part by Grants-in-Aid from the Ministry of Education, Culture, Sports, Science, and Technology of Japan (No. 17590216).

\section{References}

1 Tamargo J. Drug-induced torsade de pointes: From molecular biology to bedside. Jpn J Pharmacol. 2000;83:1-19.

2 Anderson ME, Mazur A, Yang T, Roden DM. Potassium current antagonist properties and proarrhythmic consequences of quinolone antibiotics. J Pharmacol Exp Ther. 2001;296:806810 .

3 Chiba K, Sugiyama A, Hagiwara T, Takahashi S, Takasuna K, Hashimoto K. In vivo experimental approach for the risk assessment of fluoroquinolone antibacterial agents-induced long QT syndrome. Eur J Pharmacol. 2004;486:189-200.

4 Chiba K, Sugiyama A, Satoh Y, Shiina H, Hashimoto K. Proarrhythmic effects of fluoroquinolone antibacterial agents: In vivo effects as physiologic substrate for torsades. Toxicol Appl Pharmacol. 2000;169:8-16.

5 Yap YG, Camm AJ. QT prolongation with quinolone antimicrobial agents. In: Hooper DC, Rubinstein E, editors Quinolone antimicrobial agents. 3rd ed. Washington DC: ASM Press; 2003. p. 421-440.

6 The ICH Steering Committee. The non-clinical evaluation of the potential for delayed ventricular repolarization (QT interval prolongation) by human pharmaceuticals. The International Conference on Harmonisation of Technical Requirements for Registration of Pharmaceuticals for Human Use (ICH). The 
Guideline was released for consultation under Step 4 of the ICH process in May 2005. http://www.ich.org/

7 Roden DM. Taking the "idio" out of "idiosyncratic": predicting torsades de pointes. Pacing Clin Electrophysiol. 1998;21:10291034.

8 Vos MA, van Opstal JM, Leunissen JD, Verduyn SC. Electrophysiologic parameters and predisposing factors in the generation of drug-induced Torsade de Pointes arrhythmias. Pharmacol Ther. 2001;92:109-122.

9 Kirchhof PF, Fabritz CL, Franz MR. Postrepolarization refractoriness versus conduction slowing caused by class I antiarrhythmic drugs: Antiarrhythmic and proarrhythmic effects. Circulation. 1998;97:2567-2574.

10 Sugiyama A, Hashimoto $K$. Effects of a typical $\mathrm{I}_{\mathrm{Kr}}$ channel blocker sematilide on the relationship between ventricular repolarization, refractoriness and onset of torsades de pointes. Jpn J Pharmacol. 2002;88:414-421.
11 Van de Water A, Verheyen J, Xhonneux R, Reneman RS. An improved method to correct the QT interval of the electrocardiogram for change in heart rate. J Pharmacol Methods. 1989;22:207-217.

12 Davy JM, Weissenburger J, Ertzbischoff O, Lainee P, Chezalviel F, Poirier JM, et al. Sotalol-induced torsades de pointe in the conscious dog with atrioventricular block: Role of hypokalemia. Arch Mal Coeur. 1988;81:1117-1124.

13 Duker GD, Linhardt GS. An animal model for studying class IIIinduced proarrhythmias in the halothane-anesthetized dog. J Am Coll Cardiol. 1994;23:326A.

14 Yang T, Roden DM. Extracellular potassium modulation of drug block of $\mathrm{I}_{\mathrm{Kr}}$. Implications for torsade de pointes and reverse usedependence. Circulation. 1996;93:407-411.

15 Alarcon OM, Reinosa J, Medina de Caraballo MI, Silva T. Halothane anesthesia and serum electrolytes. J Trace Elem Med Biol. 1996;10:46-49. 